\title{
Screening tools for postpartum depression: validity and cultural dimensions
}

\author{
C Zubaran 1,2, M Schumacher, M R Roxo ${ }^{3}$, K Foresti² \\ School of Medicine, University of Western Sydney, Australia \\ 2Department of Psychiatry, West Sydney Area Health Service, Australia \\ ${ }^{3}$ School of Medicine, University of Caxias do Sul, Brazil
}

\begin{abstract}
Objective: The purpose of this study is to review the main postpartum screening tools currently used in terms of their ability to screen for postnatal depression. Furthermore, the cultural characteristics of depressive postpartum symptomatology are examined. Method: A systematic literature search was conducted for the period 1987-2009, using the Medline electronic database for the following keywords: postpartum depression and postnatal depression. These terms were combined with: assessment, screening and psychometric tools. Results: Of the four screening tools reviewed and compared, the Edinburgh Postnatal Depression Scale (EPDS) and the Postpartum Depression Screening Scale (PDSS) presented substantial sensitivity and specificity as screening tools. However, none of the instruments could be rated flawless when applied to different cultural contexts. Conclusions: In addition to the EPDS, a new generation of instruments is currently available. Supplementary research is needed to substantiate their use as screening tools in general practice. Additional studies are needed to adapt and test instruments to detect postnatal depression within a wider range of languages and cultures.
\end{abstract}

Key words: Acculturation; Postnatal depression; Postpartum depression; Scales; Screening

Received: 25-05-2009

Accepted: 14-01-2010

\section{Introduction}

Mood disturbances represent the most frequent form of maternal psychiatric morbidity in the postpartum period ${ }^{1}$, and are traditionally divided into three categories: maternal sadness (postpartum blues), postpartum depression and puerperal psychosis. ${ }^{2}$ Yet there is little written about screening for these conditions within a cultural perspective.

The concept of postpartum depression as a specific diagnosis recognized by the American Psychiatric Association prompted the creation of diagnostic criteria to be listed in the Diagnostic and Statistical Manual of Mental Disorders IV (DSMIV) as referring to depression with postpartum onset that occurs during the first four weeks after birth. ${ }^{3}$ However, the definition of postpartum morbidity differs among several authors, and varies from one month to one year after birth. ${ }^{2}$

Maternal sadness affects approximately $50-80 \%$ of women

\section{Correspondence}

Carlos Zubaran

Department of Psychiatry, Blacktown Hospital, NSW 2148 Australia

email: zubaran_jr@yahoo.com in the puerperal period, with about $20 \%$ of those women developing postpartum depression. ${ }^{4}$ However, there is a wide range of reported prevalence of postpartum depression from almost $0 \%$ to almost 60\% in different countries. In some countries such as Singapore, Malta, Malaysia, Austria and Denmark there are very few reports of postpartum depressive symptoms, whereas in other countries (e.g. Brazil, Guyana, Costa Rica, Italy, Chile, South Africa, Taiwan and Korea) reported prevalence is high. ${ }^{5}$ It has been advocated that "the widely cited mean prevalence of 10-15\% for postpartum depression is not representative of the magnitude of the problem, due to the wide range of reports", which may be due to transcultural variations in reporting and interpretation of symptoms as well as distinct socio-economic variables. ${ }^{5}$

The consequences of postpartum depression affect not only the mother, but also the child and the family, and may cause matrimonial conflict and damage the child's social and cognitive development. ${ }^{4}$ Postpartum depression is infrequently diagnosed and indeed treated, despite its significant incidence and morbidity. ${ }^{6}$ The diagnosis is often masked by normal postpartum behaviors such as the mother's reactions during the adjustment period to the new physiological, social 
and behavioral conditions, including anxiety and fatigue. Moreover, the lack of clear information, as well as the lack of programs of appropriate training for the health professionals, often results in an imprecise diagnosis. ${ }^{6}$

The routine use of screening scales for the purpose of identifying symptoms of depression is an effective, simple and economical way to identify women at risk. ${ }^{8}$ Timely opportunities for routine and longitudinal screening for depression occur during the postpartum visits for maternal and pediatric re-assessment in the local health clinic., ${ }^{8,9}$ Postpartum visits are generally conducted by obstetricians, family physician, nurses and midwives, while the well-child visits, usually conducted by pediatricians and nurses. ${ }^{10}$ Evidence from several studies indicate that formal screening for depression is not only feasible in the outpatient setting but also improves rates of detection and treatment of depressive disorders, whereas informal or irregular interventions result in detection of fewer than half of cases revealed via formal and structured screening. ${ }^{10-12}$

In addition, questionnaires that are not specifically designed for the screening of postnatal depression fail to differentiate the affective from the physical symptoms and physiological changes that are typical of the puerperium, such as fatigue and changes of sleep and eating habits. ${ }^{13}$

The range and variability of validation studies undertaken with regard to the use of screening scales for postpartum depression are prolific and beg analytic interpretation before recommending their use in general (medical, midwifery or nursing) practice. In this paper commonly available scales specifically designed for screening for postpartum depression are critically analyzed. In our study, particular emphasis was given to the cultural aspects of postpartum depression.

\section{Method}

A systematic literature search was conducted using the Medline electronic database, with the following Mesh terms: postpartum depression and postnatal depression. These terms were combined with assessment, screening and psychometric tools. The literature search focused on publications from 1987 to 2009. The articles selected presented at least one topic related to maternal postpartum depression, including epidemiologic data, clinical and cultural aspects of depressive symptomatology and consequences for the offspring and the family. Additional articles specifically related to psychometric properties of screening tools were also examined.

\section{Results}

Several measures have been used for the assessment of depressive symptoms in the postpartum period. ${ }^{14}$ However, some of these instruments were not specifically designed to measure PPD. This is the case of the Beck Depression Inventory (BDI and BDI-II) ${ }^{15,16}$, the General Health Questionnaire (GHQ) ${ }^{17}$, the Zung Self-Rating Depression Scale (Zung SDS) ${ }^{18}$, the Center for Epidemiological Studies Depression Scale (CES-D) ${ }^{19}$ and the Inventory of Depressive Symptomatology (IDS). ${ }^{20}$ These are in fact instruments that measure general symptoms of depression and associated distress. In addition, the psychometric properties of some of the instruments used to measure depressive symptoms have not been completely characterized and some tools have been used infrequently. ${ }^{21}$ On the other hand, a series of other studies have tested specific screening tools to investigate depressive disorders in the postpartum period. Table I presents the different validated versions of the Edinburgh Postnatal Depression Scale (EPDS), whereas Table II presents the available versions of the Postpartum Depression Screening Scale (PDSS) in different languages.

In the postpartum period, the EPDS has been the most widely used scale to identify postpartum depression. It was developed to assist health professionals to screen community samples of postnatal mothers for depressive symptoms following childbirth and has been extensively studied and used in a number of countries due to its good sensitivity, specificity and positive predictive values. ${ }^{14}$

The PDSS is a more recent instrument also developed specifically to identify postpartum depression. A series of studies have demonstrated the reliability, construct validity, and sound psychometric properties of the PDSS, which is considered an effective instrument for identifying minor and major depression. ${ }^{54,59,60}$ The short-form version of the abovementioned questionnaire is called PDSS-SF and it consists of seven items, which were extracted from the PDSS 35-item scale, each one representing a dimension evaluated by the PDSS. ${ }^{61}$ The PDSS-SF is considered advantageous because it provides a "quick, but accurate, overall level of postpartum depression symptomatology ". 59

Finally, the Bromley Postnatal Depression Scale (BPDS) is also included here due to its specific conception and design for postpartum depression. However, there is still limited evidence on the reliability of this instrument, particularly across different cultural parameters. ${ }^{13,62}$

The specific screening tools, as mentioned above, differ conceptually from predictive inventories, which identify risk factors for postnatal depression and therefore can be used prenatally. ${ }^{63}$ There are several instruments within this class, and most of them have not been tested extensively across different cultures. This review focuses exclusively on the specific postnatal screening tools. Research evidence on the use of postpartum depression screening instruments as well as their respective psychometric data are presented here. The Transcultural usefulness of these tools are also addressed in this review.

\section{Discussion}

\section{Edinburgh Postnatal Depression Scale - EPDS}

The Edinburgh Postnatal Depression Scale (EPDS) is the most well-known and evaluated instrument for postpartum depression. ${ }^{64}$ The development of the EPDS was described firstly by Cox et al in 1987, and subsequently by Cox and Holden. ${ }^{30,65}$ The EPDS was developed firstly with 13 items, six of these being adapted from pre-existent questionnaires, and its original validation study was published in 1986. ${ }^{21}$ Later, the scale was reduced to the current 10 items and validated in a sample of 84 postpartum women. ${ }^{30}$ The scale asks the respondent about their feelings over the previous seven days. Possible responses are scored from 0 to 3, in growing order of severity, creating a maximum score of 30 . In the initial studies, the sensitivity and the specificity of the EPDS were 86\% and $78 \%$, respectively, with a positive predictive value of $73 \%$ using a cutoff point of $9 / 10$. This value is capable of detecting most cases of potential depression. ${ }^{30}$

Some of the psychometric properties of the EPDS, such as 
Table I: The available versions of the EPDS according to different languages and their respective screening properties.

\begin{tabular}{|c|c|c|c|c|c|c|}
\hline Language & Country & Cut-Off & Sensitivity (\%) & Specificity (\%) & PPV (\%) & NPV (\%) \\
\hline Amharic & Ethiopia22 & $6 / 7$ & 78.9 & 75.3 & 42.9 & 93.8 \\
\hline \multirow{3}{*}{ Chinese } & Hong Kong (China) ${ }^{23}$ & $9 / 10$ & 82 & 86 & 44 & 97 \\
\hline & Mainland China ${ }^{24}$ & 9.5 & 80 & 83 & - & - \\
\hline & Taiwan25 & $12 / 13$ & 96 & 85 & 46 & 99 \\
\hline \multirow[t]{5}{*}{ English } & Australia ${ }^{26}$ & 12.5 & 100 & 95.7 & 69.2 & 100 \\
\hline & Canada ${ }^{27}$ & 11.5 & 94 & 86 & 56 & 99 \\
\hline & Nigeria ${ }^{28}$ & 9 & 75 & 97 & 75 & 97 \\
\hline & South Africa ${ }^{29}$ & $11 / 12$ & 80 & 76 & 52.6 & 92.2 \\
\hline & United Kingdom ${ }^{30}$ & $12 / 13$ & 86 & 78 & 73 & - \\
\hline Farsi & $\operatorname{Iran}^{31}$ & $12 / 13$ & 95.3 & 87.9 & - & - \\
\hline French & France $^{32}$ & 10.5 & 80 & 92 & 91 & 97 \\
\hline German & Germany ${ }^{33}$ & 9.5 & 96 & 100 & 100 & - \\
\hline Greek & Greece ${ }^{34}$ & $8 / 9$ & 76.7 & 68.3 & 70.8 & 74.5 \\
\hline Italian & Italy ${ }^{35}$ & $9 / 10$ & 100 & 83 & 50 & 100 \\
\hline Lithuanian & Lithuania ${ }^{36}$ & 7 & 92 & 73 & 35 & 98 \\
\hline Maltese & Malta ${ }^{37}$ & $11 / 12$ & 83.3 & 96.6 & 68.2 & 98.5 \\
\hline Mongolian & Mongolia ${ }^{38}$ & $11 / 12$ & 85.7 & 71.1 & 81.4 & 77.1 \\
\hline Nepalese & Nepa|39 & 13 & 100 & 92.6 & 41.6 & - \\
\hline \multirow[t]{2}{*}{ Norwegian } & Norway ${ }^{40}$ & 10 & 100 & 87 & 60 & \\
\hline & Norway ${ }^{41}$ & 11 & 96 & 78 & 62 & - \\
\hline \multirow[t]{2}{*}{ Portuguese } & Brazil $^{42}$ & 13 & 59.5 & 88.4 & 60 & - \\
\hline & Portugal43 & 10 & 65 & 96 & 91 & 82 \\
\hline Punjabi & United Kingdom ${ }^{44}$ & 12.5 & 71.4 & 93.7 & 73 & - \\
\hline Shona & Zimbabwe ${ }^{45}$ & $11 / 12$ & 88 & 87 & 74 & 94 \\
\hline Sinhalese & Sri-Lanka ${ }^{46}$ & 9 & 90.7 & 86.8 & - & - \\
\hline \multirow[t]{4}{*}{ Spanish } & Chile ${ }^{47}$ & $9 / 10$ & 100 & 80 & 37 & - \\
\hline & Mexico 48 & $11 / 12$ & 75 & 93 & 50 & 97.6 \\
\hline & Peru 49 & 13.5 & 84.2 & 79.5 & - & - \\
\hline & Spain 50 & $10 / 11$ & 79 & 95.5 & 63.2 & 97.7 \\
\hline Swedish & Sweden ${ }^{51}$ & 11.5 & 96 & 49 & 59 & - \\
\hline Thai & Thailand 52 & $6 / 7$ & 74 & 74 & 26 & 95 \\
\hline Turkish & Turkey53 & 12.5 & 75.5 & 71.5 & 30.3 & 94.5 \\
\hline
\end{tabular}

PPV: Positive Predictive Value; NPV: Negative Predictive Value

Table II: The available versions of the PDSS according to different languages and their respective screening properties.

\begin{tabular}{|l|l|l|l|l|l|l|}
\hline Language & Country & Cut-Off & Sensitivity (\%) & Specificity (\%) & PPV (\%) & NPV (\%) \\
\hline English & Canada27 $^{27}$ & 71.5 & 94 & 59 & 31 & 98 \\
Spanish & United States $^{54}$ & 80 & 94 & 98 & 90 & 99 \\
Portuguese & United States $^{55}$ & 60 & 84 & 84 & 75 & 90 \\
\multirow{2}{*}{ Thai } & Brazil56 $^{\text {Brazil57 }}$ & 102 & 94 & 95 & 75 & 99 \\
\hline & Thailand58 & 51 & 72 & 79 & 23 & 97 \\
\end{tabular}

PPV: Positive Predictive Value; NPV: Negative Predictive Value

its specificity and sensitivity, have been tested extensively among various cultures in different countries. The scale has been tested in countries as diverse as England, Australia, Sweden, Chile, Canada, Portugal, Italy, France, China, South Africa, Brazil, Spain, Turkey and Germany. ${ }^{66}$ The sensitivity observed in the validation studies presented variations ranging from $65 \%$ to $100 \%$, while the specificity presented variations from $49 \%$ to $100 \%$. The great variability of results among the different studies was due to variations in

methodology used, cut-off points, diagnostic criteria and in the period between childbirth and the moment of application of the instrument 66

Studies developed in United Kingdom have demonstrated that using a cutoff point of $12 / 13$, in the sixth week of the postpartum period, the EPDS shows a sensitivity from 68 to $95 \%$ and a specificity of $78 \%$ to $96 \%$, when compared to the diagnosis of major depression through psychiatric interview. ${ }^{30,67}$ The cutoff point of 9/10, used to increase the sensitivity of the instrument, demonstrates a sensitivity of 84 to $100 \%$, as well as specificity of $82 \%$ to $88 \% .{ }^{67} \mathrm{~A}$ total score 
higher than 12 in the postpartum period indicates a larger probability of depression, but it does not supply a measure of the severity of the symptoms. ${ }^{68}$ Matthey et al. investigated the increasing use in the literature of non-validated cutoff scores of the EPDS, as well as different wording and formatting in the scale. ${ }^{69}$ It was advocated in this study that the scale should be worded and formatted as originally described by its authors, which includes the validated score of 13 or more and of 15 or more when reporting a probable depressive episode in English-speaking women during postnatal and antenatal periods, respectively. ${ }^{69}$ However, different cutoff scores may be warranted for different cultural groups. ${ }^{14}$

Some researchers suggest that mothers with increased risk of developing postpartum depression can be identified early in the postpartum period, which would assist secondary prevention. ${ }^{64}$ Dennis et al. demonstrated that the EPDS, when applied with cutoff points of 9/10 during the first week of the postpartum period, has satisfactory discriminative and prognostic power to distinguish the mothers with increased risk of developing depression during the period between the fourth and eighth weeks. ${ }^{1}$ The author suggests that there is a strong connection to maternal mood during the three appraised weeks and women with symptoms of major or minor depression in the first week of postpartum have a larger probability of developing a mental disorder in the subsequent weeks. The results make possible the implementation of secondary prevention for later postpartum depression. ${ }^{1}$ On the other hand, Lee et al. demonstrated that when the EPDS is used in the first 48 hours after the child's birth, the psychometric properties of this instrument show a significant number of false-positive results. ${ }^{64}$

The EPDS measures emotional symptoms of postpartum depression and does not evaluate somatic symptoms, except for one item that measures sleep difficulties. ${ }^{14}$ The main limitations of EPDS is that this instrument does not evaluate the context of a women's experience as a new mother, and features such as loss of control, loneliness, unrealness, irritability, loss of self and concentration difficulties. ${ }^{14}$ Moreover, appetite loss, weight reduction and reduced libido, symptoms that can happen due to a depressive episode, are not detected by EPDS. Postnatal depression with predominant features of psychomotor retardation seems also not to be detected by this scale, and could be particularly important for studies about the impact of maternal depression on the mother-baby interaction. ${ }^{70}$

Although EPDS is a screening instrument and not a diagnostic one, it is often used as an assessment/diagnostic tool in clinical practice. The application of the EPDS should not be a substitute for a psychiatric evaluation. A clinical interview is essential for diagnosis, since the scale does not provide a measure of severity of the distress, but only indicates the probability of postpartum depression. ${ }^{71}$

\section{Postpartum Depression Screening Scale - PDSS}

The PDSS is a self-rating scale recently developed and conceptually based on a series of qualitative data collected and analyzed in previous research by Beck. ${ }^{72-74}$ This instrument is not a diagnostic tool and was developed to overcome some of the EPDS limitations cited above and evaluate more introspective concerns of a woman in the postpartum period. ${ }^{56}$ Seven conceptual domains are included in the PDSS: sleep and appetite disturbance; anxiety/insecurity; emotional lability; cognitive impairment; loss of self-esteem; guilt/shame and suicidal thoughts. Each domain is composed of five identifying symptoms that the mothers can present with in the period after childbirth. These are itemized in degree of intensity, that can vary from full disagreement to full agreement using a 5-point Likert scale; scores range from 35 to 175, and relate to how women were feeling in the preceding two weeks. ${ }^{60}$ The PDSS is available in English ${ }^{60}$, Spanish ${ }^{59}$, Turkish ${ }^{75}$, Thai ${ }^{58}$ and Portuguese versions. ${ }^{56,57}$ The original English version of the PDSS has also been tested among Native American English ${ }^{76}$ and First Nations and Métis women. ${ }^{27}$

In the first PDSS validation study ${ }^{60}, 525$ mothers were recruited during the first 6 weeks of the postpartum period to test the reliability and the validity of the new instrument. Reliability analysis identified Cronbach's alpha indexes higher than 0.80 for each of the seven domains. The PDSS was filled out initially by the mothers, and followed by a Structured Clinical Interview (undertaken by clinicians 'blinded' to the score) based on DSM-IV to confirm, or not, the correlation. A cutoff point of 80 was proposed for major postpartum depression, with sensitivity of $94 \%$ and specificity of $98 \%$ and a cutoff point of 60 for major or minor depressive distress, with sensitivity and specificity of $91 \%$ and 72\%, respectively, using the Receiver Operator Curve (ROC). In an additional study ${ }^{54}$, to strengthen the findings, Beck and Gable investigated a further 150 mothers in the sixth week of postpartum period. According to this study, a cutoff value of 70 can cause a reduced number (6\%) of falsenegative results. However, up to $16 \%$ of the mothers in a non-depressive state will have a false positive result.

The PDSS can also be applied in its brief version that consists of the first seven items of the full scale. These items demonstrate a high correlation with the seven dimensions that are used in the longer version $(r=0.91)$. The obtained scores range from 7 to 35 and present similar levels of reliability and validity when compared to the complete PDSS. ${ }^{11}$ Using the Flesch index (1948), a score of 91.9 was achieved in ease of reading by the PDSS, which is consistent with "very easy" comprehension. ${ }^{77}$ In addition to this favorable characteristic of the PDSS, further comparison with the EPDS demonstrated reliability and validity. The PDSS offers specific information on the interviewee's clinical status in each one of the seven appraised domains. Such references are useful for the doctor, midwife or nurse, since they inform on the mother's current state in the specifically appraised areas and the clinician can target specific and appropriate therapeutic interventions to meet individual needs. In a study that compared the PDSS with the EPDS and with the Beck Depression Inventory (BDI-II) ${ }^{78}$, the PDSS was the only instrument of the three instruments studied which evaluated all the five initial symptoms of postpartum depression anxiety, insomnia, agitation, irritability and confusion. ${ }^{77}$ Furthermore, loss of self-esteem was only measured by the PDSS. This scale is the one which has the highest Combination of sensitivity and specificity to ( $94 \%$ and $98 \%$, respectively) when using 80 as the cutoff point for major depression. ${ }^{77}$ 


\section{The Bromley Postnatal Depression Scale - BPDS}

The Bromley Postnatal Depression Scale (BPDS) was developed in 1992 in the United Kingdom, and is an instrument specifically designed for detecting the presence of both current and previous episodes of postnatal depression. ${ }^{79}$ It allows women to report their mood and behaviors during pregnancy and the postpartum period for the current and all previous births. It has 10 items as well as a chart to indicate when the postpartum depression began, how long it lasted and when it was the worst for all births. These characteristics make this instrument unique, because there is a possibility for establishing a longitudinal course of postpartum depression. ${ }^{14}$ It is mainly designed to detect previous and current episodes of PND, as well as, appropriate for use in postal surveys. ${ }^{13}$

There is limited information about the psychometric properties of the BPDS. This instrument has not been validated transculturally either. In a community sample, this scale demonstrated good test-retest reliability, but the internal consistency has not been established. ${ }^{14} \mathrm{~A}$ study conducted with a sample of 165 women revealed that the sensitivity and specificity of the BPDS were respectively $62 \%$ and $94 \% .{ }^{13}$ Although the BPDS was considered by its proponents to "reliable", the analysis of reliability of this questionnaire was established via test-retest ratings only. ${ }^{13}$ The lower sensitivity of the BPDS may be related to the fact that this instrument also detects cases of less severe forms of depressive and anxiety disorders. ${ }^{13}$

\section{The cultural contours of depressive postpartum symptomatology}

It has been observed that factors such as socio-economic status, biological factors, and stigma associated with mental health disorders may influence expression of depressive symptoms and prevalence rates of postpartum depression. ${ }^{80}$ Contrary to the theories postulated by Stern and Kruckman (1983), who advocated that PPD was infrequent or absent in non-Western cultures, recent evidence has demonstrated that PPD is a transcultural phenomenon. ${ }^{81}$ Despite the fact that postnatal depression appears to be a universal condition with apparently similar rates in different countries, anthropologists question the cross-cultural equivalence of depression, particularly at a life stage so influenced by cultural factors. ${ }^{82}$

From an anthropological perspective, postpartum depression has been considered a "culture-bound syndrome", since major changes in family structure that occur in Western and secularized societies may result in increased rates of depression. ${ }^{83}$ On the other hand, medical professionals advocate that the similarity of postpartum depression symptoms and continuous rates across the cultures would indicate a common biological diathesis. Although biological factors appear to play a central role in explaining the homogenous prevalence trends of postnatal depression worldwide, cultural forces and socio-economic elements play a pivotal role in the way that maternal distress is manifested during the postpartum period. ${ }^{84}$

A series of international projects aimed at the transcultural understanding of perinatal depressive phenomena confirmed the transcultural nature of PPD. 5,82,85,86,87,88 However, transcultural comparative studies of depressive symptomatology also demonstrated that symptoms tend to vary according to specific cultural backgrounds. ${ }^{89}$ Results from a study conducted in nine countries not only corroborated that postpartum symptomatology is manifested across different cultural boundaries but also indicated that depressive symptoms can be even more prominent among non-Western women. ${ }^{87}$

Members of non-Western cultures tend to express specific depressive symptoms such as somatization, which contrasts with common "Western world" symptoms such as sadness and guilty feelings. ${ }^{90} \mathrm{~A}$ study designed to compare postnatal depression in the United Kingdom and Taiwan ${ }^{91}$ obtained similar prevalence rates of postnatal depression, although British women reported a better quality of care during the postnatal period, which appears to favor biological determinants for the manifestation of this disorder. Although specific cultural factors may mold the expression of distress through predominantly physical or psychological manifestations, recent evidence indicates that both forms of manifestation are significantly correlated. ${ }^{57}$

The "transcultural approach" to postnatal mental disorders might help to clarify "the ways in which social factors can influence the evolution of psychopathology". 84 In addition, culturally validated methodologies are available for comparative research into postnatal depression among different health systems and countries. ${ }^{88}$ The Western-non-Western dichotomy is no longer considered an adequate divide to account for the clinical and epidemiological variations of PPD. ${ }^{81}$ The significant variation in the prevalence figures of PPD according to studies conducted in different non-Western countries suggests that the way PPD presents itself cannot be matched transculturally by a stationary diagnostic criteria established according to Western yardsticks. ${ }^{81}$

\section{The worldwide prevalence variations of postpartum depression}

There is a consensus among practitioners that postpartum depression may advance unrecognized ${ }^{92}$, despite the fact that depressive symptomatology reported by women in the postpartum period can be collected across diverse countries and regions. ${ }^{87}$ The wide variability of postnatal depression suggests that the proposed prevalence rates of 10 to $15 \%$ in the general population may underestimate the actual magnitude of the disorder. The majority of studies that investigated PPD have been developed in developed countries (Affonso, 2000 \#64) and the most commonly used screening instruments for postnatal depression were conceived and developed in the developed Western world. In fact, there is worldwide evidence that postnatal depression and prevalence figures vary according to different countries even when the same screening instrument is used. ${ }^{90}$

A literature review on prevalence of postpartum depression and depressive symptoms in 40 countries revealed that the prevalence of $\mathrm{PPD}$, measured mostly by the Edinburgh Postpartum Depression Scale, ranged from 
almost 0\% to almost 60\%. ${ }^{5}$ In another international study, a set of research instruments were tested in eight countries in order to investigate the cross-cultural equivalence of postnatal depression. ${ }^{88}$ Although a state of morbid postpartum sadness comparable to postnatal depression was reported in all countries, there was no consensus on the understanding of this process as an illness that requires a categorical treatment approach. ${ }^{82}$ In addition, the rates of postpartum depression may fluctuate according to native or immigrant status, since acculturation stress appears to be conducive to more prominent postpartum difficulties. ${ }^{90}$

A study designed to investigate the prevalence of depressive symptoms in two Latin American countries, Costa Rica and Chile ${ }^{93}$, observed that, albeit with different socio-demographic measures, rates of depression were comparable in developing countries of Latin America as well. In Brazil, among women of low income, 43\% presented with at least one depressive episode during the first six months after birth and the prevalence of depressive episodes in the third month was $12 \% .^{94,95}$ In Brazilian women, depressive symptoms are common during pregnancy and are associated with indicators of socio-economic deprivation, violence and the loss of an intimate relationship. ${ }^{96}$ Nonetheless, Brazilian studies examining this issue indicate a prevalence of postpartum depression similar to the international average approximately 13\%.66,94 A Brazilian study about maternity blues ${ }^{97}$ demonstrated a postpartum symptom peak occurrence on the fifth day, with symptoms more significantly associated with overemotionalism and oversensitivity.

Asian countries differ considerably in terms of philosophical traditions, religious practices, cultural life and general attitudes toward physical and psychological problems. ${ }^{98}$ As the world's largest and most populous continent, Asia encompasses members of the Arab world in Western Asia to Eastern capitalist economies, such as Japan and Korea. A recent review which examined research conducted in 17 Asian countries revealed that the prevalence of PPD in Asian countries ranged from 3.5\% to 63.3\%, having Malaysia and Pakistan the lowest and highest prevalence respectively. ${ }^{98}$

The risk factors for postpartum depression in Asian cultures were categorized in five main groups: physical/biological, psychological, obstetric/pediatric, socio-demographic and cultural factors. ${ }^{98}$ Although Arab women also receive familial support in the postpartum period, as it is the case for Asian women, the reported prevalence rates of PPD are comparable to those reported in Western countries. ${ }^{81}$ Marital difficulties, polygamy, previous psychiatric history, first child, and pediatric illnesses have been reported as the most common psychosocial factors associated with PPD in the United Arab Emirates. ${ }^{99}$

After Asia, Africa is the world's second largest and most populous continent. Africa's population is relatively young and in some African states almost half of the population is under 25 years of age. The burden imposed on the African people through the spread of infectious diseases and frequent tribal and military conflicts aggravate further the impact of mental disorders on women. The challenges associated with women reproductive health in the West African subregion, including Sexually Transmitted Diseases (STDs), especially HIV/ AIDS, and the high maternal mortality, which is associated with insufficient provision of health care and associated resources, adds to the burden of psychiatric complications faced by women during the life cycle, particularly PPD. ${ }^{100}$ In Uganda, PPD was associated with negative life events, including unplanned pregnancy and physical illness of both mother and newborn. ${ }^{101}$ Research conducted in a South African periurban settlement revealed elevated rates of PPD (34.7\%), being maternal depression in that community associated with deprived engagement with the partner and child. ${ }^{102}$ In Morocco, an Arab African country, maternal depressive disorders were also associated with marital conflicts and stressful life events during pregnancy, including health problems of the infant. ${ }^{103}$

In fact, in some cases, as described in a descriptive report in Nigeria, folklore beliefs and intense family involvement with maternal affairs may cause additional stress and be a reason for delay in the referral process to appropriate medical care. ${ }^{104}$ The prevalence rate of PPD among Nigerian women has been reported around 14\% rate at 6 to 8 weeks postpartum and psychosocial factors appear significantly related to the genesis this morbidity. ${ }^{105}$ The gender of thee baby has also been linked to PPPD I Nigeria. ${ }^{106}$ The prevalence of maternity blues was reported to be $31.3 \%$, being strong predictor of this disorder single mothers ${ }^{107}$ and obstetrics complications. ${ }^{108}$

A recent study that investigated PPD in the Democratic Republic of Congo reported that, despite the fact that Western models of psychopathology may be inappropriate for establishing diagnosis according to local tenets, a local syndrome similar to depression was observed. ${ }^{109}$ The shortage of psychiatrists in West Africa combined with of limited screening skills of the obstetric personnel hinders the detection and effective treatment of PPD in Africa. ${ }^{100}$ Nevertheless, a research conducted in Zimbabwe demonstrated the feasibility and effectiveness of a brief method for identifying women at high risk for developing a postnatal mental disorder. ${ }^{110}$

In summary, although postpartum depression is a disorder of worldwide occurrence, there are regional variations in the prevalence rates as well as the symptomatic expressions of PPD. The prevalence of PDD in a given society appears to be influenced by a myriad of local and environmental factors, including cultural and folklore elements, religious practices, socioeconomic deprivation, lack of health care infrastructure and poor public health indicators. The EPDS has been the most widely screening tool, whereas some other methodologies are starting to be used as screening alternatives.

\section{Conclusion}

Since there is a high prevalence of mood disturbance during the postpartum period, as well as potentially harmful consequences to the mother and the child, and the marital relationship, it is important that the postpartum dysphorias are correctly identified). The early diagnosis of postpartum depression makes possible the implementation of preventive measures to avoid the 
progression of the disease into a more serious form, as well as strategies to assist in the development of the mother-baby relationship.

This current review has provided information about some of the main assessment instruments of postpartum depression currently available. Several other scales and diagnostic tools not specifically developed for postpartum depression can be used in the evaluation of risk, albeit with smaller degrees of sensitivity than the instruments described here. The use of scales such as EPDS, PDSS and PDPI should be strongly recommended, since their simplicity, low cost, and high sensitivity in the detection of mothers with postpartum depression make them useful when used correctly. All the postpartum and well-child visits should be considered potential opportunities to screen for postpartum depression, therefore appropriate education and training of health care providers (obstetricians, family physicians, nurse midwives, family nurse practitioners, pediatricians), in conjunction with psychiatric services, should be provided to increase awareness of this problem. However, practitioners need to be aware of the current research concerning the reliability and validity of these instruments when applied to their own cultural and social environments.

The fact that all the screening scales for postpartum depression have been developed based on the English language generates complications of cultural relevance, semantics, sensitivity, concepts and constructs. The different dialects within countries also raises the possibility of idiosyncratic elements, since each population or community has an individual way of interpreting maternal depressive symptoms This fact indicates the need for the development of assessment instruments for postpartum depression that are validated within various cultures.

\section{Acknowledgments}

We kindly thank Dr. Gill White, Mrs. Andrea Pavan, Dr. Enda Bannan and Ms. Laura Borrelli for their valuable assistance during distinct phases of this study. This study was partially supported by grant \# 000605-25.00/03-8 from the Secretaria de Ciência e Tecnologia do Estado do Rio Grande do Sul, Brazil.

\section{References}

1. Dennis C. Can we identify mothers at risk for postpartum depression in the immediate postpartum period using the Edinburgh Postnatal Depression Scale? J Affect Disord 2004; 78: 1639.

2. Foresti $K$, Zubaran CA. Disforia em período puerperal: evidências científicas e tendências conceituais. Revista de Ciências Médicas 2004; 2: 16-21

3. American Psychiatric Association. Diagnostic and Statistical Manual of Mental Disorders. Fourth Editon, Text Revision ed. Whashington, DC: American Psychiatric Association; 2000.

4. Miller LJ. Pospartum depression. JAMA 2002; 287: 762-6

5. Halbreich U, Alarcon RD, Calil H, Douki S, Gaszner P, Jadresic E, et al. Culturally-sensitive complaints of depressions and anxieties in women. J Affect Disord 2006.

6. Lee DT, Yip AS, Chiu HF, Chung TK. Screening for Postnatal Depression Using the Double-Test Strategy. Psychosom Med 2000; 62: $258-63$

7. Epperson CN. Postpartum major depression: detection and treatment. Am Fam Physician 1999: 59: 2247-54.

8. Gjerdingen DK, Yawn BP. Postpartum Depression Screening: Importance, Methods, Barriers, and Recommendations for Practice. J Am Board Fam Med 2007; 20: 280-88.

9. Mancini F, Carlson C, Albers L. Use of the Postpartum Depression Screening Scale in a collaborative obstetric practice

.J Midwifery Womens Health 2007; 52: 429-34.

10. Chaudron LH, Szilagyi PG, Kitzman HJ, Wadkins H, Conwell Y. Detection of Postpartum Depressive Symptoms by Screening at Well-Child Visits. Pediatrics 2004; 113: 551-8.

11. Evins GG, Theofrastous JP, Galvin SL. Postpartum depression: A comparison of screening and routine clinical evaluation. Am J Obstet Gynecol 2000; 182: 1080-2.

12. Georgiopoulos AM, Bryan TL, Wollan P, Yawn BP. Routine screening for postpartum depression. J Fam Pract 2001; 50: 11 1-22.

13. Stein G, Van den Akker O. The retrospective diagnosis of postnatal depression by questionnaire. J Psychosom Res 1992; 36(1): 67-75.

14. Boyd RC, Le HN, Somberg R. Review of screening instruments for postpartum depression. Arch Women Ment Health 2005; 8: 141-53.

15. Beck AT, Ward CH, Mendelson M, Mock J, Erbaugh J. An inventory for measuring depression. Arch Gen Psychiatry 1961; 4: 561-71.

16. Beck AT, Steer R, Brown G. Manual for Beck Depression Inventory II (BDI-II). San Antonio, TX: Psychology Corporation; 1996

17. Goldberg D. Manual of the General Health Questionnaire. Windsor, England: NFER Publishing; 1978.

18. Zung W. A self-rating depression scale. Arch Gen Psychiatry 1965; 12: $63-70$

19. Radloff $L$. The CES-D scale: A self report depression scale for research in the general population. Applied Psychological Measurement 1977; 1: 385-401.

20. Rush AJ, Gullion CM, Basco MR, Jarrett RB, Trivedi $M H$. The Inventory of Depressive Symptomatology (IDS): psychometric properties. Psychol Med 1996; 26(3): 477-86.

21. Cox J. Postnatal depression: A guide for health professionals. Edinburgh: Churchill Livingstone; 1986.

22. Tesfaye $M$, Hanlon $C$, Wondimagegn D, Alem A. Detecting postnatal common mental disorders in Addis Ababa, Ethiopia: Validation of the Edinburgh Postnatal Depression Scale and Kessler Scales. J Affect Disord 2009

23. Lee DT, Yip SK, Chiu HF, Leung TY, Chan KP, Chau IO, et al. Detecting postnatal depression in Chinese women. Validation of the Chinese version of the Edinburgh Postnatal Depression Scale. Br J Psychiatry 1998; 172: 433-7.

24. Wang Y, Guo X, Lau Y, Chan KS, Yin L, Chen J. Psychometric evaluation of the Mainland Chinese version of the Edinburgh Postnatal Depression Scale. Int J Nurs Stud 2009; 46(6): 813-23.

25. Teng HW, Hsu CS, Shih SM, Lu ML, Pan JJ, Shen WW. Screening postpartum depression with the Taiwanese version of the Edinburgh Postnatal Depression scale. Compr Psychiatry 2005; 46(4): 261-5.

26. Boyce P, Stubbs J, Todd A. The Edinburgh Postnatal Depression Scale: validation for an Australian sample. Aust N Z J Psychiatry 1993; 27(3) 472-6.

27. Clarke PJ. Validation of two postpartum depression screening scales with a sample of First Nations and Metis women. Can J Nurs Res 2008; 40(1): 113-25.

28. Uwakwe R, Okonkwo JE. Affective (depressive) morbidity in puerperal Nigerian women: validation of the Edinburgh Postnatal Depression Scale. Acta Psychiatr Scand 2003; 107(4): 251 -9.

29. Lawrie TA, Hofmeyr GJ, de Jager M, Berk M. Validation of the Edinburgh Postnatal Depression Scale on a cohort of South African women. S Afr Med J 1998; 88(10): 1340-4. 
30. Cox J, Holden J, Sagovsky R. Detection of postnatal depression: development of the 10-item Edinburgh Postnatal Depression Scale. Br J Psychiatry 1987; 150: 782-6.

31. Mazhari S, Nakhaee N. Validation of the Edinburgh Postnatal Depression Scale in an Iranian sample. Arch Womens Ment Health 2007; 10(6): 293-7.

32. Guedeney N, Fermanian J. Validation study of the French version of the Edinburgh Postnatal Depression Scale (EPDS): new results about use and psychometric properties. Eur Psychiatry 1998; 13(2): 83-9.

33. Bergant AM, Nguyen T, Heim K, Ulmer H, Dapunt O. [German language version and validation of the Edinburgh postnatal depression scale]. Dtsch Med Wochenschr 1998; 123(3): 35-40.

34. Vivilaki VG, Dafermos V, Kogevinas M, Bitsios P, Lionis C. The Edinburgh Postnatal Depression Scale: translation and validation for a Greek sample. BMC Public Health 2009; 9: 329.

35. Carpiniello B, Pariante CM, Serri F, Costa G, Carta MG. Validation of the Edinburgh Postnatal Depression Scale in Italy.J Psychosom Obstet Gynaecol 1997; 18(4): 280-5.

36. Bunevicius A, Kusminskas L, Bunevicius R. Validation of the Lithuanian version of the Edinburgh Postnatal Depression Scale. Medicina (Kaunas) 2009; 45(7): 544-8.

37. Felice E, Saliba J, Grech V, Cox J. Validation of the Maltese version of the Edinburgh Postnatal Depression Scale. Arch Womens Ment Health 2006; 9(2): 75-80.

38. Pollock JI, Manaseki-Holland S, Patel V. Detection of depression in women of child-bearing age in non-Western cultures: a comparison of the Edinburgh Postnatal Depression Scale and the Self-Reporting Questionnaire-20 in Mongolia. J Affect Disord 2006; 92(2-3): 267-71.

39. Regmi S, Sligl W, Carter D, Grut W, Seear M. A controlled study of postpartum depression among Nepalese women: validation of the Edinburgh Postpartum Depression Scale in Kathmandu. Trop Med Int Health 2002; 7(4): 378-82.

40. Eberhard-Gran M, Eskild A, Tambs K, Schei B, Opjordsmoen S. The Edinburgh Postnatal Depression Scale: validation in a Norwegian community sample. Nord J Psychiatry 2001; 55(2): 113-7.

41. Berle JO, Aarre TF, Mykletun A, Dahl AA, Holsten F. Screening for postnatal depression. Validation of the Norwegian version of the Edinburgh Postnatal Depression Scale, and assessment of risk factors for postnatal depression. J Affect Disord 2003; 76(1-3): 151-6.

42. Santos IS, Matijasevich A, Tavares BF, Barros AJ, Botelho IP, Lapolli C, et al. Validation of the Edinburgh Postnatal Depression Scale (EPDS) in a sample of mothers from the 2004 Pelotas Birth Cohort Study. Cad Saude Publica 2007; 23(11): 2577-88.

43. Areias ME, Kumar R, Barros H, Figueiredo B. Correlates of postnatal depression in mothers and fathers. Br J Psychiatry 1996; 169: 36-41.

44. Werrett J, Clifford C. Validation of the Punjabi version of the Edinburgh postnatal depression scale (EPDS). Int J Nurs Stud 2006; 43(2): 227-36.

45. Chibanda D, Mangezi W, Tshimanga M, Woelk G, Rusakaniko P, Stranix-Chibanda L, et al. Validation of the Edinburgh Postnatal Depression Scale among women in a high HIV prevalence area in urban Zimbabwe. Arch Womens Ment Health 2009.

46. Rowel D, Jayawardena P, Fernando N. Validation of the Sinhala translation of Edinburgh Postnatal Depression Scale. Ceylon Med J 2008; 53(1): 10-3.

47. Jadresic E, Araya R, Jara C. Validation of the Edinburgh Postnatal Depression Scale (EPDS) in Chilean postpartum women. J Psychosom Obstet Gynaecol 1995; 16(4): 187-91.

48. Alvarado-Esquivel C, Sifuentes-Alvarez A, Salas-Martinez C, Martinez-Garcia S. Validation of the Edinburgh Postpartum Depression Scale in a population of puerperal women in Mexico.
Clin Pract Epidemol Ment Health 2006; 2: 33 .

49. Vega-Dienstmaier JM, Mazzotti Suarez G, Campos Sanchez M. [Validation of a Spanish version of the Edinburgh Postnatal Depression Scale]. Actas Esp Psiquiatr 2002; 30(2): 106-11.

50. Garcia-Esteve L, Ascaso C, Ojuel J, Navarro P. Validation of the Edinburgh Postnatal Depression Scale (EPDS) in Spanish mothers. J Affect Disord 2003; 75(1): 71-6

51. Wickberg B, Hwang CP. The Edinburgh Postnatal Depression Scale: validation on a Swedish community sample. Acta Psychiatr Scand. 1996; 94(3): 181-4.

52. Pitanupong J, Liabsuetrakul T, Vittayanont A. Validation of the Thai Edinburgh Postnatal Depression Scale for screening postpartum depression. Psychiatry Res 2007; 149(1-3): 253-9.

53. Aydin N, Inandi T, Yigit A, Hodoglugil NN. Validation of the Turkish version of the Edinburgh Postnatal Depression Scale among women within their first postpartum year. Soc Psychiatry Psychiatr Epidemiol 2004; 39(6): 483-6.

54. Beck CT, Gable RK. Further Validation of the Postpartum Depression Screening Scale. Nurs Res 2001; 50: 155-64.

55. Beck CT, Gable RK. Screening performance of the postpartum depression screening scale--Spanish version. J Transcult Nurs 2005; 16(4): 331-8.

56. Cantilino A, Carvalho JA, Maia A, Albuquerque C, Cantilino G, Sougey EB. Translation, Validation and Cultural Aspects of Postpartum Depression Screening Scale in Brazilian Portuguese. Transcult Psychiatry 2007; 44: 672-84.

57. Zubaran C, Foresti K, Schumacher M, Amoretti A, Muller L, Thorell M, et al. Validation of a screening instrument for postpartum depression in Southern Brazil. J Psychosom Obstet Gynaecol 2009.

58. Vittayanont A, Liabsuetrakul T, Pitanupong J. Development of Postpartum Depression Screening Scale (PDSS): a Thai version for screening postpartum depression.J Med Assoc Thai 2006; 89(1): 1-7.

59. Beck CT, Gable RK. Postpartum Depression Screening Scale Spanish Version. Nurs Res 2003; 52: 296-306.

60. Beck CT, Gable RK. Postpartum Depression Screening Scale: Development and psychometric testing. Nurs Res 2000; 49: 272-82.

61. Beck CT, Gable RK. Postpartum Depression Screening Scale Manual. Los Angeles: Western Psychological Services; 2002.

62. McLaren L, Kuh D, Hardy R, Mishra G. Postnatal depression and the original mother-child relationship: a prospective cohort study. J Affect Disord 2007; 100(1-3): 211-9.

63. Beck CT, Records K, Rice M. Further development of the Postpartum Depression Predictors Inventory-Revised. J Obstet Gynecol Neonatal Nurs 2006; 35: 735-45.

64. Lee DT, Yip AS, Chan SSM, Tsui MHY, Wong WS, Chung TKH. Postdelivery Screening for Postpartum Depression. Psychosom Med 2003; 65: 357-61.

65. Holden J. Using the Edinburgh Post Natal Depression Scale in Clinical Practice. In: Cox J, Holden JM, editors. Perinatal Psychiatry: Use and Misuse of the Edinburgh Postnatal Depression Scale. London: Gaskell; 1994. p. 54-81.

66. Eberhard-Gran M, Eskild A, Tambs K, Opjordsmoen S, Samuelsen SO. Review of validation studies of the Edinburgh Postnatal Depression Scale. Acta Psychiatr Scand 2001; 104: 243-9.

67. Murray L, Carothers AD. The validation of the EPDS on a community sample. Br J Psychiatry 1990; 157: 288-90.

68. Pope S. Postnatal depression: A systematic review of published scientific literature to 1999. Camberra: National Health and Medical Research Council. 2000.

69. Matthey S, Henshaw C, Elliott S, Barnett B. Variability in use of cut-off scores and formats on the Edinburgh Postnatal Depression Scale- 
implications for clinical and research practice. Arch Womens Ment Health 2006; 9: 309-15.

70. Guedeney N, Fermanian J, Guelfi JD, Kumar RC. The Edinburgh Postnatal Depression Scale (EPDS) and the detection of major depressive disorders in early postpartum: some concerns about false negatives. J Affect Disord 2000; 61: 107-12.

71. Hanna B, Jarman H, Savage S. The clinical application of three screening tools for recognizing post-partum depression. Int $J$ Nurs Pract 2004; 10: 72-9

72. Beck CT. The lived experiences of postpartum depression: $A$ phenomenological study. Nurs Res 1992; 41: 166-70.

73. Beck CT. Teetering on the edge: A substantive theory of postpartum depression. Nurs Res 1993; 42: 42-8.

74. Beck CT. Postpartum depressed mothers' experiences interacting with their children. Nurs Res 1996; 45: 98-104.

75. Karacam Z, Kitis Y. [The Postpartum Depression Screening Scale: its reliability and validity for the Turkish population]. Turk Psikiyatri Derg 2008; 19(2): 187-96.

76. Baker L, Cross S, Greaver L, Wei G, Lewis R. Prevalence of postpartum depression in a native American population. Matern Child Health J 2005; 9(1): 21-5.

77. Beck CT, Gable RK. Comparative Analysis of the Performance of the Postpartum Depression Screening Scale With Two Other Depression Instruments. Nurs Res 2001; 50: 242-50.

78. Beck AT, Steer RA, Brown GK. BDI-II Manual. San Antonio: The Psychological Corporation; 1996.

79. Stein G, Van den Akker O. The retrospective diagnosis of postnatal depression by questionnaire. J Psychosom Res 1992; 36: 67-75.

80. Halbreich U, Karkun S. Cross-cultural and social diversity of prevalence of postpartum depression and depressive symptoms. $J$ Affect Disord 2006; 91: 97-111.

81. Posmontier B, Horowitz JA. Postpartum practices and depression prevalences: technocentric and ethnokinship cultural perspectives. $J$ Transcult Nurs 2004; 15(1): 34-43.

82. Oates MR, Cox JL, Neema S, Asten P, Glangeaud-Freudenthal N, Figueiredo B, et al. Postnatal depression across countries and cultures: a qualitative study. Br J Psychiatry Suppl 2004; 46: s1 0-6.

83. Stern G, Kruckman L. Multi-disciplinary perspectives on postpartum depression: an anthropological critique. Soc Sci Med 1983; 17: 1027-41.

84. Kumar R. Postnatal mental illness: a transcultural perspective. Soc Psychiatry Psychiatr Epidemiol 1994; 29: 250-64.

85. Bernazzani O, Conroy S, Marks MN, Siddle KA, Guedeney N, Bifulco $A$, et al. Contextual Assessment of the Maternity Experience: development of an instrument for cross-cultural research. Br J Psychiatry Suppl 2004; 46: s24-30.

86. Gorman LL, O'Hara MW, Figueiredo B, Hayes S, Jacquemain F, Kammerer MH, et al. Adaptation of the structured clinical interview for DSM-IV disorders for assessing depression in women during pregnancy and post-partum across countries and cultures. Br J Psychiatry Suppl 2004; 46: s17-23.

87. Affonso DD, De AK, Horowitz JA, Mayberry LJ. An international study exploring levels of postpartum depressive symptomatology. J Psychosom Res 2000; 49: 207-16.

88. Asten P, Marks MN, Oates MR. Aims, measures, study sites and participant samples of the Transcultural Study of Postnatal Depression. Br J Psychiatry Suppl 2004; 46: s3-9.

89. Jablensky A, Sartorius N, Gulbinat W, Ernberg G. Characteristics of depressive patients contacting psychiatric services in four cultures. A report from the who collaborative study on the assessment of depressive disorders. Acta Psychiatr Scand 1981; 63: 367-83.
90. Bashiri N, Spielvogel AM. Postpartum depression: a cross-cultural perspective. Primary Care Update for OB/GYNS. 1999; 6(3): 82-7.

91. Huang YC, Mathers N. Postnatal depression -- biological or cultural? A comparative study of postnatal women in the UK and Taiwan. J AdV Nurs 2001; 33: 279-87.

92. Bashiri N, Spielvogel AM. Postpartum depression: a cross cultural perspective. Psychiatry update 1999; 6: 82-7.

93. Wolf AW, De Andraca I, Lozoff B. Maternal depression in three Latin American samples. Soc Psychiatry Psychiatr Epidemiol 2002; 37: 169-76.

94. Da Silva VA, Santos AR, Carvalho MS, Martins ML, Teixeira NA. Prenatal and postnatal depression among low income Brazilian women. Brz J Med Biol Res 1998; 31: 799-804.

95. Faisal-Cury A, Tedesco JJ, Kahhale S, Menezes PR, Zugaib M. Postpartum depression: in relation to life events and patterns of coping. Arch Women Ment Health 2004; 7: 123-31.

96. Lovisi GM, Lopez JR, Coutinho ES, Patel V. Poverty, violence and depression during pregnancy: a survey of mothers attending a public hospital in Brazil. Psychol Med 2005; 35: 1485-92.

97. Rohde LA, Busnello E, Wolf A, Zomer A, Shansis F, Martins S, et al. Maternity blues in Brazilian women Acta Psychiatr Scand 1997; 95: 231-5.

98. Klainin P, Arthur DG. Postpartum depression in Asian cultures: a literature review. Int J Nurs Stud 2009; 46(10): 1355-73.

99. Abou-Saleh M. Postpartum psychiatric illness in Arab culture. Arab Journal of Psychiatry 1997; 8(1): 1-5.

100. Aina OF. An overview of the socio-cultural and psychiatric aspects of women's reproductive health in West Africa. Niger Postgrad Med J 2007; 14(3): 231-7.

101. Nakku JE, Nakasi G, Mirembe F. Postpartum major depression at six weeks in primary health care: prevalence and associated factors. Afr Health Sci 2006; 6(4): 207-14.

102. Cooper PJ, Tomlinson M, Swartz L, Woolgar M, Murray L, Molteno C. Post-partum depression and the mother-infant relationship in a South African peri-urban settlement. Br J Psychiatry 1999; 175: 5548.

103. Agoub M, Moussaoui D, Battas O. Prevalence of postpartum depression in a Moroccan sample. Arch Womens Ment Health 2005; 8(1): 37-43.

104. Okafor CB. Folklore linked to pregnancy and birth in Nigeria. West J Nurs Res 2000; 22(2): 189-202.

105. Aderibigbe YA, Gureje O, Omigbodun O. Postnatal emotional disorders in Nigerian women. A study of antecedents and associations. Br J Psychiatry 1993; 163: 645-50.

106. Fatoye FO, Adeyemi AB, Oladimeji BY. Postpartum depression following normal vaginal delivery among Nigerian women. Psychol Rep 2004; 94(3 Pt 2): 1276-8.

107. Adewuya AO. The maternity blues in Western Nigerian women: prevalence and risk factors. Am J Obstet Gynecol 2005; 193(4): $1522-5$.

108. Adewuya AO, Fatoye FO, Ola BA, Ijaodola OR, Ibigbami SM. Sociodemographic and obstetric risk factors for postpartum depressive symptoms in Nigerian women. J Psychiatr Pract 2005; $11(5): 353-8$

109. Bass JK, Ryder RW, Lammers MC, Mukaba TN, Bolton PA. Postpartum depression in Kinshasa, Democratic Republic of Congo: validation of a concept using a mixed-methods cross-cultural approach. Trop Med Int Health 2008; 13(12): 1534-42.

110. Nhiwatiwa S, Patel V, Acuda W. Predicting postnatal mental disorder with a screening questionnaire: a prospective cohort study from Zimbabwe. J Epidemiol Community Health 1998; 52(4): 262-6. 